\title{
Characterization of Ultrafiltration Membranes
}

\author{
Shoji Kimura \\ Department of Chemical Engineering, The University of Tokyo, \\ 7-3-1 Hongo, Bunkyo-ku, Tokyo 113, Japan
}

(Received December 14, 1990)

\begin{abstract}
Transport phenomena, that are relevant to ultrafiltration membranes, are discussed. Their basic characteristics are given by the pore-flow theory. In actual applications, however, their behaviors are controlled by gel layer formation, osmotic pressure of solutes, adsorption or plugging in pores and by other chemical and physical natures of membranes used. Also electric charges give rise important effects both on flux and solute separation. All these behaviors need careful analysis of data taken by model and actual solutions. Also recently distinction between RO and UF membranes becomes obscure and their relation should be made more clear. These results will finally be combined to establish methods to select proper membrane materials and to design proper membrane modules for actual applications.

KEY WORDS UTtrafiltration / Concentration Polarization / Pore-flow Theory / Protein Adsorption / Charged Membrane /
\end{abstract}

\section{INTRODUCTION}

Ultrafiltration membranes (UF) are defined that their pore sizes are smaller than those of microfiltration membranes (MF), whose pore sizes are about 0.01 micro-meter at the smallest. Usually pores in UF membranes can not be measured by any physical method, and are expressed by molecular weight cutoff (MWCO) values. The smallest MWCO value of UF membranes is about 1,000, and then molecules smaller than this are separated by reverse osmosis (RO) membranes. Conceptionally UF membranes separate molecules, while MF membranes separate particles. UF membranes separate solute molecules by sieving mechanism, while RO membranes separate molecules not by their sizes, but by molecular interactions between solutes, solvents and membrane materials. All these distinctions, however, are becoming obscure and more works should be done to clarify transport phenomena in these membranes. In the following transport phenomena in UF membranes will be focused and research results on transport phenomena obtained so far will be explained.

\section{Transport Phenomena in UF}

There are two important transport phenomena in general membrane permeation processes, namely, concentration polarization near membrane surfaces and transport inside membranes. These are shown in Fig. 1, where it is seen that a membrane separates a solution of higher concentration $\left(C_{m}\right)$ than a bulk solution $\left(C_{b}\right)$ due to concentration polarization phenomena, whose relation is given by $\mathrm{Eq} .1$. To reduce this effect it is necessary to increase a mass transfer coefficient ( $k$ ) 


$$
\begin{aligned}
& \frac{C_{m}-C_{p}}{C_{b}-C_{p}}=\exp \left(J_{v} / k\right) \\
& k=D / \delta
\end{aligned}
$$

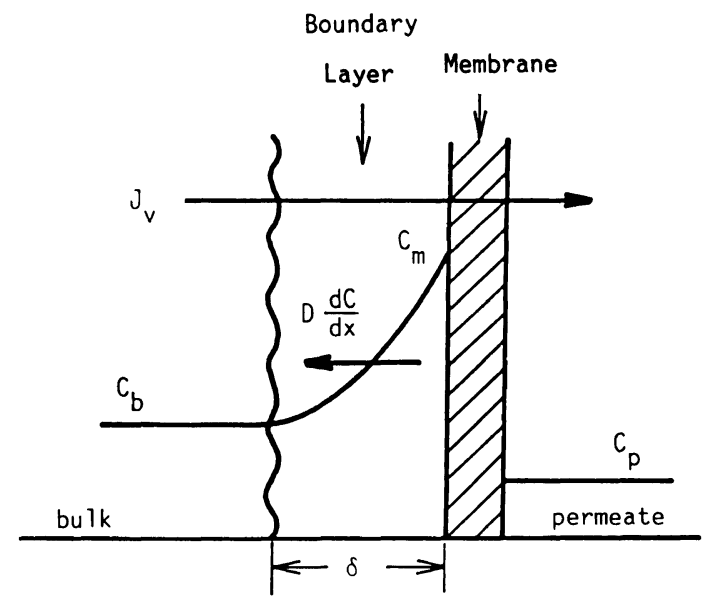

Fig. 1 Concentration polarization phenomena.

at a membrane surface, which is defined as a diffusion coefficient of a solute (D) divided by a boundary layer thickness, $\delta$, as is given by Eq. 2. Since molecular weight of solutes treated by UF is large and their diffusion coefficients are smal1, a concentration at a membrane surface tends to increase and sometimes becomes larger than a gelling or saturation concentration, which results in a gel or deposit layer formation on a membrane surface. This may lead to severe fouling of membranes.

Transport processes inside membrane have been analyzed by the theory based on the thermodynamics of irreversible processes ${ }^{2}$ and practical equations generally used are those developed by Spiegler and Kedem ${ }^{3}$ and given as follows.

$$
\begin{aligned}
& J_{v}=L_{p} \cdot(\Delta P-\Delta \pi) \\
& R=\frac{\sigma(1-F)}{1-\sigma F}
\end{aligned}
$$

, where

$$
F=\exp \left(-\frac{1-\sigma}{P} J_{v}\right)
$$

and $R$ is real rejection of a membrane defined as

$$
R=\left(C_{m}-C_{p}\right) / C_{m}
$$

$L_{p}, \sigma$ and $P$ are solution permeability, reflection coefficient and solute permeability, respectively. To determine these transport coefficients from experimentally obtained data, usually a curve-fitting method is adopted and an example is shown in Fig. 2, where $R$ is plotted against $1 / J_{V}$ to show that when $J_{y} \rightarrow \infty R \rightarrow \sigma$. Although these parameters are experimentally determined they can not give transport mechanism inside a membrane. 
Transport phenomena inside UF membrane is usually explained by the pore-flow theory ${ }^{4}$, which assumes solute molecules as a sphere having radius $r_{s}$ and it flow through a cylindrical straight pore with radius $r_{p}$ in a membrane. In this case all phenomena is determined by the ratio of $r_{s}$ to $r_{p}$, which is defined as $q$, and transport parameters, such as $L_{p}, \sigma$, and $P$ are given as follows.

$$
\begin{aligned}
& L_{p}=r_{p}^{2} / 8_{\mu} \cdot A_{k} / \Delta x \\
& \sigma=1-g(q) \cdot S_{F} \\
& P=D \cdot f(q) \cdot S_{D} A_{k} / \Delta x
\end{aligned}
$$

, where $A_{k} / \Delta x$ is a ratio of the total cross-sectional pore area to the effective membrane area divided by a pore length. $S_{D}$ and $S_{F}$ are steric hindrance factors for diffusion flow and filtration flow, respectively, and given as follows. $f(q)$ and $g(q)$ are wall correction factors for diffusion flow and filtration flow, respectively, and given as follows.

$$
\begin{aligned}
& S_{D}=(1-q)^{2} \\
& S_{F}=2(1-q)^{2}-(1-q)^{4} \\
& f(q)=\frac{1-2.1 q+2.1 q^{3}-1.7 q^{5}+0.73 q^{6}}{1-0.76 q^{5}} \\
& g(q)=\frac{1-(2 / 3) q^{2}-0.2 q^{5}}{1-0.76 q^{5}}
\end{aligned}
$$

Since it is difficult to measure $r_{p}$ directly, it is estimated from experimentally determined $q$ using $r_{s}$, which is obtained from diffusion coefficients using the following Stokes-Einstein equation.

$$
r_{s}=k T / 6 \pi \mu D
$$

So far data show good agreement with the above theory, when $f(q)$ and $\mathrm{g}(\mathrm{q})$ are taken as $1^{5}$. An example is shown in Fig. 3. Further experimental confirmation is necessary to check the validity of the pore-flow theory.

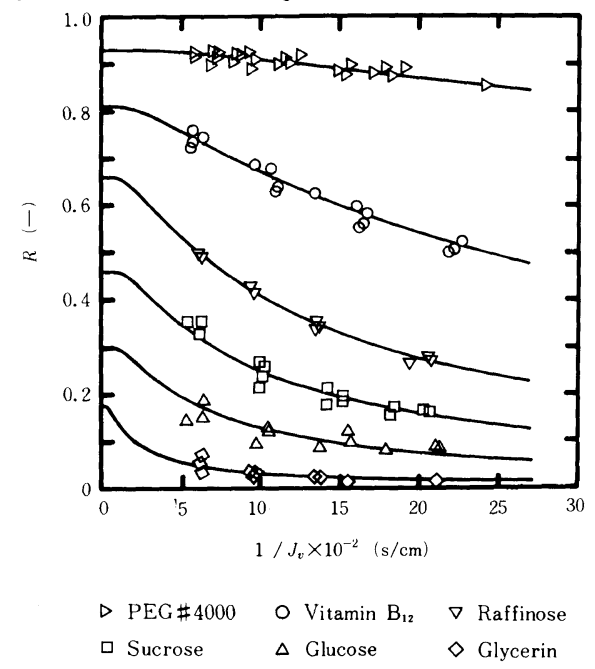

Fig. 2 R vs. $1 / J_{v}$.

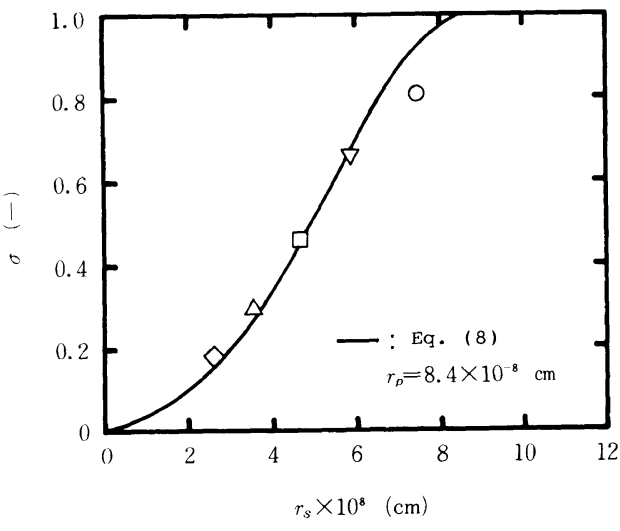

Fig. 3 Reflection coefficient. The line is given by Eq. (8), when $f(q) \& g(q)$ are 1 . 


\section{Limiting Flux}

Another important phenomena in UF is its limiting flux problem, that is, flux is not proportional to applied pressure and reaches to a constant value, which is called a limiting flux. Usually this value is not so large and limits the capacity of UF equipments. To understand the mechanism of flux limitation and to find ways to increase this value are very important for promoting actual applications of the UF process.

The first theoretical explanation is a gel polarization model, which assumes a gel layer formed on a membrane surface has large resistance to permeation and the increase of pressure merely results in the increase of gel layer resistance, that is proportional to its thickness. Usually this model is given by the next equation, which is transformed from Eq. (1).

$$
J_{1 i m}=k \cdot \ln \left(C_{g}-C_{p}\right) /\left(C_{b}-C_{p}\right) \simeq k \cdot \ln \left(C_{g} / C_{b}\right)
$$

, where $C_{g}$ is the concentration of gel layer, which is constant, merely depends on solutes and not on operating conditions. $C_{g}$ can be obtained from experimental data by plotting $\mathrm{J}$ im against $\mathrm{lnC}_{\mathrm{b}}$ and extrapolating J lim to zero. But usually values thus obtained are not large enough to show gelation behavior and this is one of reasons this gel polarization model is not supported by researchers. Another reason is that its relation between the resistance model is not clear, that is expressed as

$$
J_{v}=\Delta P /\left(R_{m}+R_{g}\right)
$$

, where $R_{m}$ and $R_{g}$ are resistance of membrane and gel layer, respectively. The relation between Eqs. (15) and (16) is not clear.

The next model is a osmotic pressure mode ${ }^{6}$, which assumes that osmotic pressure increase due to the concentration polarization limits the flux. The osmotic pressure predicted by the van't Hoff equation is not large for dilute solution of high molecular solutes, but at larger concentration the osmotic pressure increases very rapidly as shown in Fig. 5 . This model can be easily expressed by using equations as follows.

$$
\begin{aligned}
& J_{v}=(\Delta P-\Delta \pi) / R_{m} \\
& \begin{aligned}
\Delta \pi & =\pi\left(C_{m}\right)-\pi\left(C_{p}\right) \\
& =\pi\left(C_{m}\right) \\
\pi(C) & =a C^{n} \\
J_{v} & =k \cdot \ln \left(C_{m} / C_{b}\right)
\end{aligned}
\end{aligned}
$$

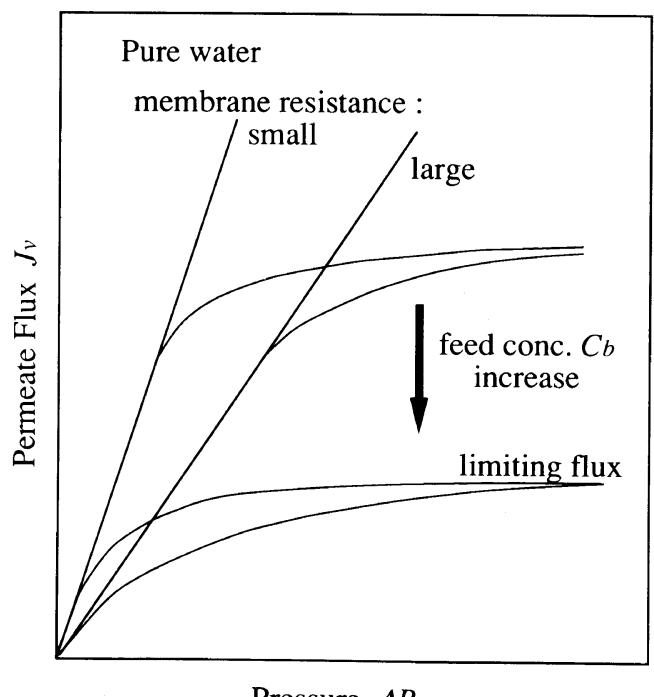

Pressure $\Delta P$

Fig. 4 Limiting flux behavior of UF membranes.

By combining above equations $J_{v}$ can be obtained. 
Since this model does not include any uncertain parameter as $C$ and osmotic pressure can be directly measured, it can predict flux behavior more easily and precisely. There is another theory, which is called a boundary layer resistance mode ${ }^{7}$, and assumes existence of the flux resistance at a boundary layer of a membrane surface. This theory is a little bit complicated but it is finally proved that it is equivalent to the osmotic pressure model.

One more important factor is adsorption of solutes at a surface or inside of pores in a membrane. Many data have shown that flux is dependent on membrane materials, even if the same feed solution is used, and this has been considered as the difference of adsorption of membrane materials. An example is shown in Fig. 6. Here various resistances of flux in 3 kinds of membrane materials are measured against ovalbumin concentration and it shows adsorption in PAN membrane is large. So this effect can not be ignored and should be
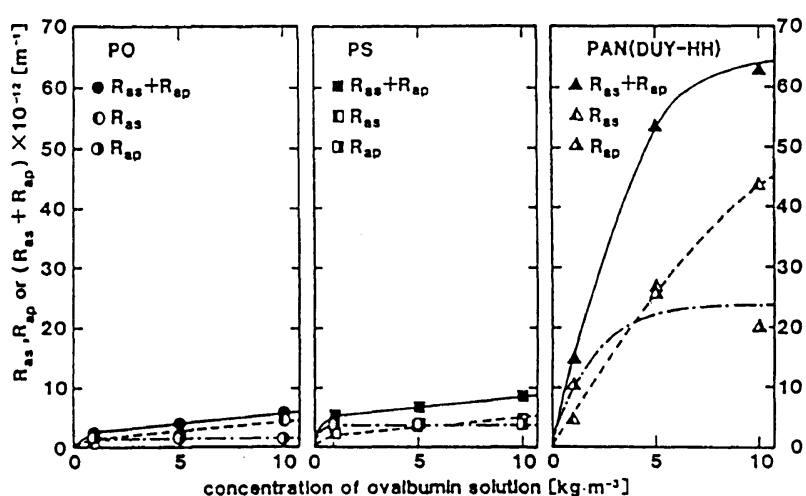

Fig. 6 Resistances caused by Adsorption on surface $\left(R_{a s}\right)$ taken into consideration for the prediction of UF membranes. An example of this trial is shown in Fig. 7. In this case $L_{p}$ is replaced by $L_{p}{ }^{\prime}$, which is given as follows.

$$
L_{p}{ }^{\prime}=\frac{1}{R_{m}+R_{a}}
$$

- where $R_{a}$ is the resistance due to adsorption, which is determined from Fig. 6. Then $J_{v}$ is given as

$$
J_{v}=L_{p}{ }^{\prime}\left(\Delta P-\Delta \pi\left(C_{m}\right)\right)
$$

Fig. 7 shows good agreement of this model. The adsorption mechanism of proteins to membranes is still difficult to understand and in the meantime this kind of treatment is useful to predict membrane behavior.

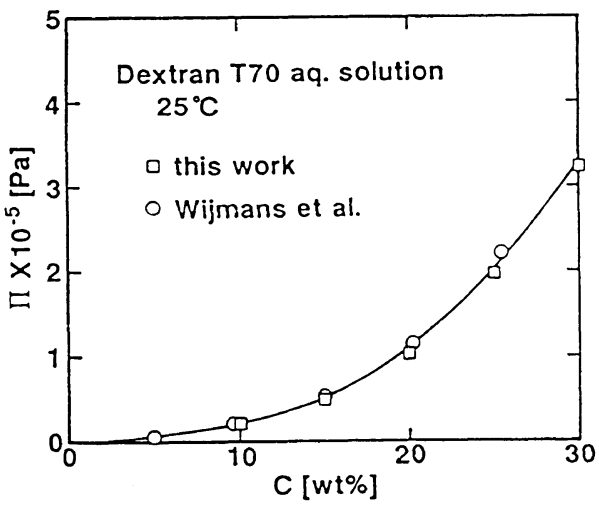

Fig. 5 Osmotic pressure of dextran $T 70$.

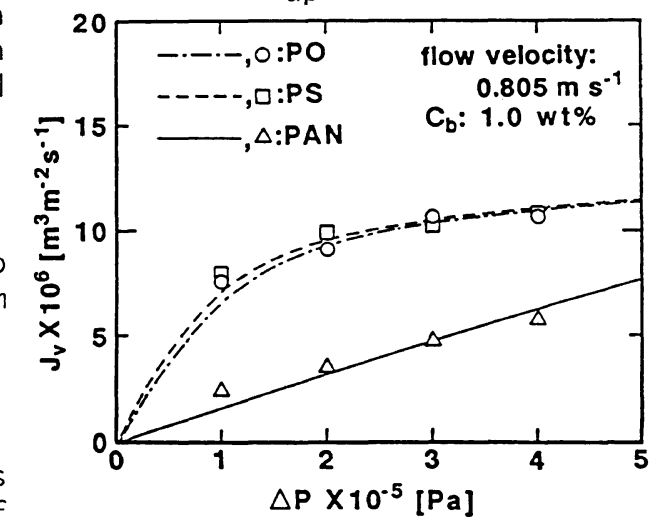

Fig. 7 Experimental and calculated flux for three kinds of membranes. 


\section{Charged Ultrafiltration Membranes}

UF membranes made of polymers that have fixed charged group can behave as charged membranes, which can reject charged solutes by Donnan exclusion. Such membrane can reject electrolytes, whose molecular weights are smaller than MWCO values of membranes, or it can separate electrolytes from non-electrolytes 8,9 . Typical materials for such membrane are sulfonated polysulfone (SPS) and polysulfone with ammonium group (APS). These membranes have been commercialized and used for removal of trace amount of ions or colored substances from various water and effluents.

An interesting example is separation of aminoacids using such membranes 10 . Their dissociated forms are dependent on $\mathrm{pH}$ and rejection dependence of aminoacids corresponds with them. An example is shown in Fig. 8, where reflection coefficients match perfectly with dissociated forms. And at low concentrations interaction between aminoacids is small and so aminoacids having different isoelectric points can be separated by adjusting solution $\mathrm{pH}$. This is demonstrated in Fig. 9, where sodium L-aspartate is rejected, while L-isoleucine is not by a SPS membrane.

Another example is separation of proteins 11 , but in this case separation is not so easy as before, because for this purpose membranes that have large MWCO values are necessary, and at the same time configuration changes of protein molecules occur by changing $\mathrm{pH}$ and thereby adsorption tendency also changes. In Fig. 10 separation of myoglobin (M.W.:17,500, Isoelectric point: $\mathrm{pH} 7.0$ ) and cytochrome C (M.W.:12,400, Isoelectric point: pH: 9.0) using SPS membrane is demonstrated. But separation of BSA (M.W.:69,000, Isoelectric point: $\mathrm{pH}$ 4.8) from other proteins was not successful, because BSA tends to adsorb to SPS membranes and change their MWCO values as shown in Fig. 11.<smiles>CCCCC(C)(C)c1ccc(Oc2ccc(S(=O)(=O)c3ccc(OCCCC(C)(C)C)cc3)cc2)cc1</smiles>

$S P S$

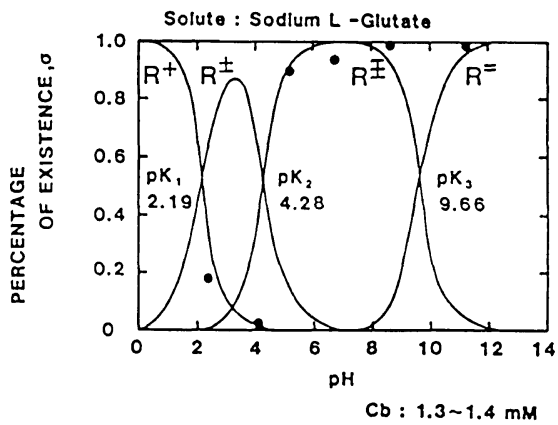

Fig. 8 Relation between reflection coefficient and dissociation of sodium L-glutamate.

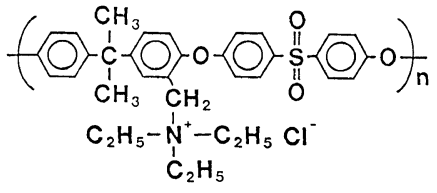

\section{A P S}

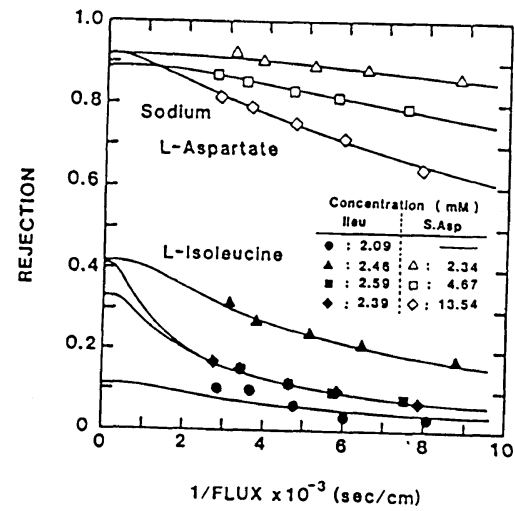

Fig. 9 Rejection from a mixed solution of sodium L-aspartate and $\mathrm{L}-i$ soleucine. 


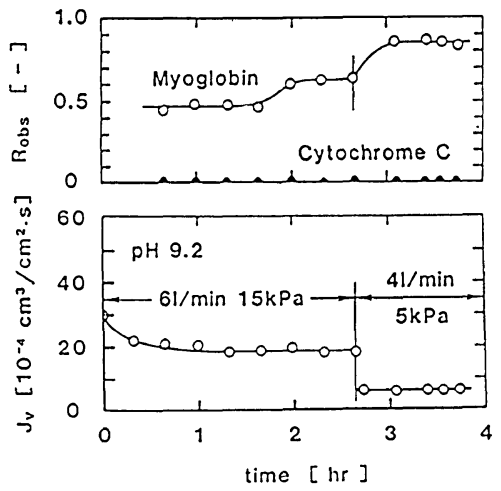

Fig. 10 Separation of mixed myoglobin and cytochrome $\mathrm{C}$ by a SPS membrane.

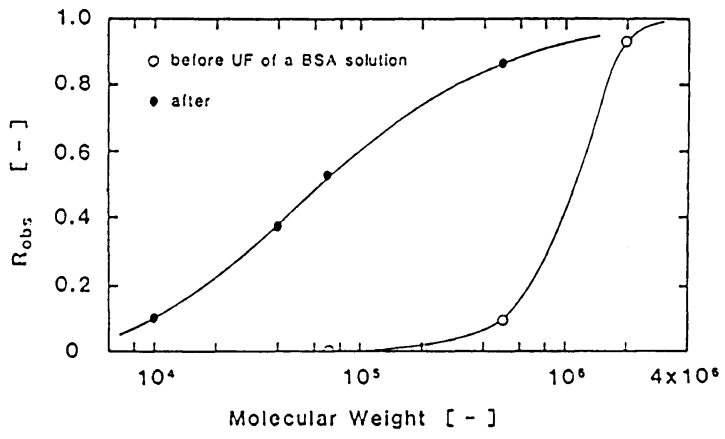

Fig. 11 MWCO value of a SPS membrane measured with dextran

\section{Inorganic Membranes}

Recently many inorganic membranes have been commercialized as UF and MF membranes and are competing with organic membranes in many actual applications. Inorganic membranes are usually resistant to high temperature and can be sterilized by steam, and this is favorable for them to be applied in various biotechnological processes.

Also many inorganic materials, such as alumina, zirconia, carbon and porous glass have different adsorption nature to proteins and some membrane materials claim less fouling compared to polymeric membranes. One of the methods to characterize such membrane is zeta potential ${ }^{12}$. An example is shown in Fig. 12, where results of alumina, silica and SiC are shown. Also their surface can be modified by treatments to have different zeta potential, which is shown in Fig. 13, where original alumina (A) is modified with sulfophenyl group (N) and with amino group (C). These membranes are expected to behave differently against

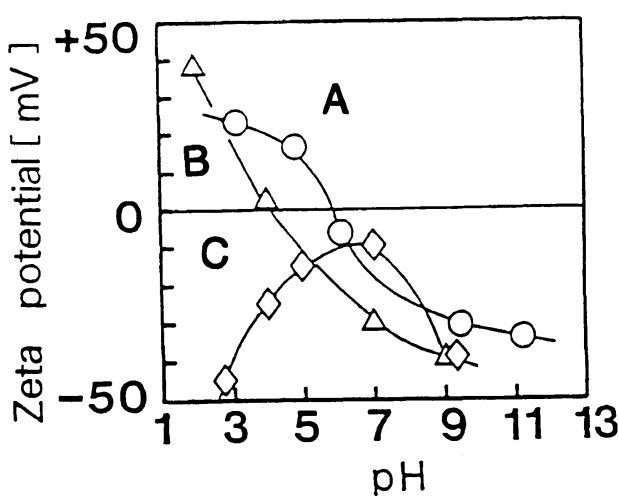

Fig. 12 Zeta potential of ceramic membranes. A: Alumina, B: Porous glass, C: SiC. (Pore Size: $0.5 \mathrm{~m}$ )

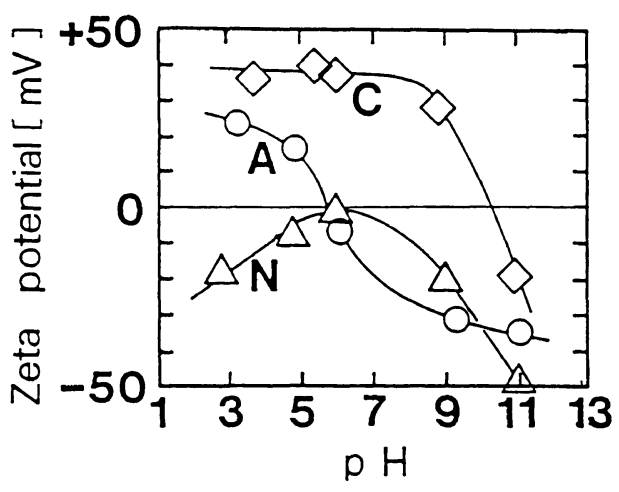

Fig. 13 Zeta potential of alumina having different groups.

A: Alumina, N: Sulfonyl group, C: Amino group 
adsorption and fouling, but still compilation of data is necessary to get conclusion.

Durability of inorganic membranes to high temperature can be demonstrated by the other example ${ }^{3}$. Usually effect of temperature on transport property of UF membrane can be predicted by changes of viscosity, diffusion coefficient and osmotic pressure of solutions. This is shown in Fig. 14, where changes of permeability of alumina membrane are shown for various solutes and dotted lines are predicted values from data at $20^{\circ} \mathrm{C}$. But for polysulfone membranes, whose results are shown in Fig. 15, permeability becomes larger that that predicted by ordinary temperature correction and this shows the expansion of pores in a SPS membrane by increasing temperature. This shows again the stability of inorganic membranes.

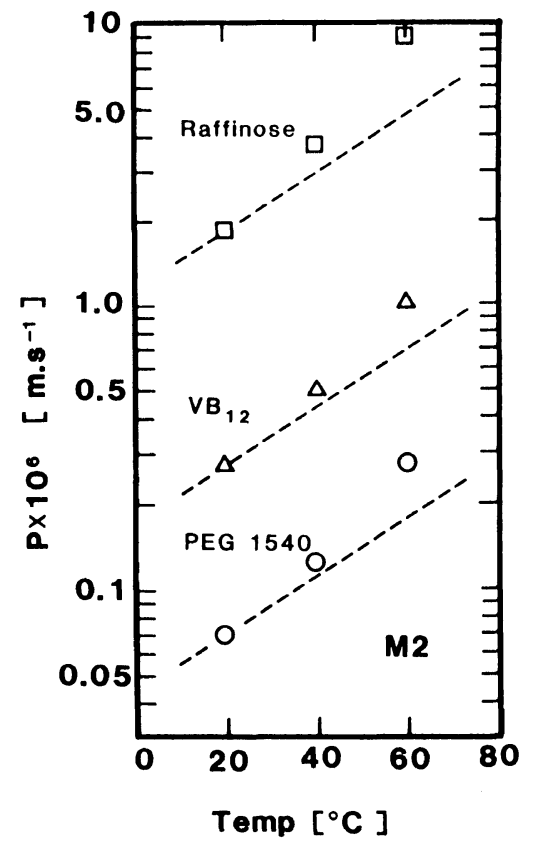

Fig. 14 Effect of temperature on permeability of alumina memb.

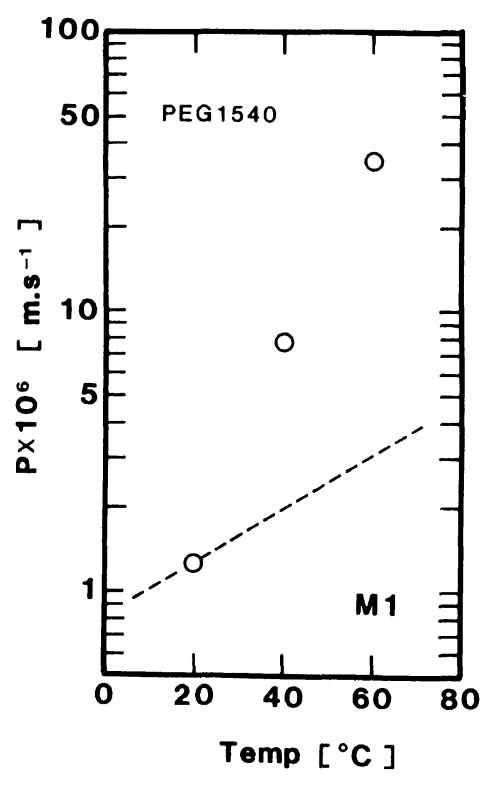

Fig. 15 Effect of temperature on permeability of SPS membrane

\section{Low Pressure RO Membranes}

Extensive use of RO membrane in production of ultra-pure water in the electronic industry for cleaning IC products forced to develop various RO membranes used at lower pressure than in seawater desalination. Recently requirements for these membranes becomes more and more severe and their rejection should be good not only for inorganic salts, but also for organic solutes, such as 2-propanol. As a result RO membranes are approaching to UF membranes and their distinction is becoming more obscure. Also many charged RO membranes are used for such a purpose.

Transport phenomena of charged membrane can be treated by the same Eqs. (3) to(5) to obtain their transport paraineters, but the pore-flow theory can no more be applicable and a new theoretical treatrient is necessary. But historically the Nernst-Planck equation has been used for 
analysis of charged membranes. However, since this treatment does not includes volume flow through membrane, its modification is necessary. This is called a extended Nernst-Planck equation. Its solution and relation between the Kedem-Spiegler treatment have been extensively studied $^{14}$.

Deen et a ${ }^{15}$ tried to combine the Nernst-Planck equation with the pore-flow theory and succeeded to analyze permeation rates of dextran, dextran sulfate and diethylaminoethyl dextran through glomerular membranes, which is considered as a negatively charged membrane. Application of this kind of analysis for low pressure RO membrane behavior will be interesting.

\section{REFERENCES}

1. S. Nakao, T. Nomura and S. Kimura, AIChEJ., 25, 615 (1979)

2. A. Katchalsky and P. F. Curran, "Nonequilibrium Thermodynamics in Biophysics", Harvard Univ. Press, Cambridge, Mass., 1965, Chap. 11.

3. K. S. Spiegler and O. Kedem, Desalination, 1311 (1965)

4. A. Verniory, R. Du Bois, P. Decoodt, J. P. Gassee and P. P. Lambert, J. Gen. Physiol., 62439 (1973)

5. S. Nakao and S. Kimura, J. Chem. Eng. Japan, 15200 (1982)

6. J. G. Wijmans, S. Wakao and C. À. Snolders, J. Memb. Sci., 20 115 (1984)

7. J. G. Wijmans, S. Nakao, J. W. A. van den Berg, F. R. Troelstra and C. A. Smolders, J. Hemb. Sci., 22117 (1985)

8. I. Jitsuhara and S. Kimura, J. Chein. Eng. Japan, 16389 (1933)

9. I. Jitsuhara and S. Kimura, J. Chein. Eng. Japan, 16394 (1983)

10. S. Kimura and A. Tamano, "lembranes and Membrane Processes" ed. by E. Drioli and i. Nakagaki, Plenum Pub. Corp., 1986, p.191.

11. S. Nakao, H. Osada, H. Kurata, T. Tsuru and S. Kimura, Desalination, 70191 (1988)

12. Y. Shimizu, MAKU(Membrane), 15179 (1990)

13. T. Nomura, S. Nakao and S. Kiriura, Kagaku Kougaku Ronbunshu, 13811 (1987)

14. T. Tsuru, S. Nakao and S. Kimura, J. Chem. Eng. Japan, 23604 (1990)

15. W. M. Deen, B. Satvat and J. M. Jamieson, Am. J. Physiol. 38 F126 (1980) 